\title{
Percepción del riesgo, comportamientos riesgosos y seroprevalencia del VIH en trabajadoras sexuales de Georgetown, Guyana1
}

\author{
Keith H. Carter, ${ }^{2}$ Bhiro P. Harry, ${ }^{3}$ Michael Jeune ${ }^{4} y$ \\ Devian Nicholson ${ }^{3}$
}

RESUMEN En abril de 1993 se realizó un estudio en 108 trabajadoras sexuales que ejercían la prostitución en Georgetown, Guyana. Mediante entrevistas y pruebas de sangre, se investigaron las relaciones entre la seroprevalencia del VIH y conocimientos sobre el sida, prácticas riesgosas, características de los clientes y uso de condones. Las mujeres que trabajaban en la calle -a diferencia de las que trabajaban en bares, hoteles y Puerto Georgetown-solian cobrar menos, estaban en peor situación socioeconómica y tenían clientes en circunstancias similares; por lo tanto, estas mujeres se clasificaron dentro de un estrato socioeconómico "inferior" y las restantes dentro de uno "superior". La seroprevalencia general del VIH observada en las trabajadoras sexuales fue de $25 \%$ (IC95\% = 17 a 33\%). Las 50 mujeres del estrato inferior tuvieron, sin embargo, una seroprevalencia relativamente alta (42\% en comparación con $10 \%$ de las del estrato superior), y a ese grupo pertenecían 21 de las 27 mujeres seropositivas al VIH. El origen declarado de los clientes (guyaneses o extranjeros), la disposición de las trabajadoras a tener relaciones sexuales sin condón y el uso de condones por los clientes variaron según el estrato. Las participantes del estrato más alto estaban más dispuestas a tener relaciones sexuales sin condón y en ambos estratos las mujeres sabían poco sobre la causa del sida y la transmisión del VIH. Muchas trabajadoras declararon haber contraído una enfermedad de transmisión sexual (ETS) en los 2 años anteriores o haber consumido drogas ilícitas. Según los datos recogidos, los clientes guyaneses usan condones con menos frecuencia que los extranjeros, fenómeno que implica un mayor riesgo de contraer el VIH por contacto con un guyanés o de transmitirlo a un guyanés. La seroprevalencia del VIH en trabajadoras que dijeron tener solo clientes guyaneses fue estadísticamente mayor que la observada en las que declararon que sus clientes eran solo extranjeros. La seroprevalencia del VIH en mujeres que declararon tener más de cinco clientes a la semana fue estadísticamente mayor que en las que tenían menos clientes. La seropositividad al VIH fue relativamente frecuente entre las 12 trabajadoras que declararon haber usado cocaina. En general, los resultados confirman la necesidad de fortalecer las intervenciones dirigidas a las trabajadoras sexuales y a sus clientes $y$, particularmente, de hacer un esfuerzo concertado para intensificar la promoción, distribución y comercialización social de los condones; mejorar los servicios de atención de ETS que ofrezcan tratamiento y orientación a las trabajadoras sexuales, y ampliar las actividades educativas dirigidas a sus clientes guyaneses.

1 Se publicó en inglés en esta revista, 1997, Vol. 1, No. 6, con el título "HIV risk perception, risk behavior, and seroprevalence among female commercial sex workers in Georgetown, Guyana".

2 Organización Panamericana de la Salud, Georgetown, Guyana. Dirección postal: Pan American Health Organization, Lot 8 Brickdam, Georgetown, Guyana.

3 Hospital Público, Georgetown, Guyana.

4 Oficial de Comunicaciones, Georgetown, Guyana.
Según la Secretaría Nacional del Sida de Guyana, en el período de 1987 a 1992, $280(71,8 \%)$ de los 390 pacientes de sida confirmados en Guyana eran hombres, y de ellos, un mínimo de $59 \%$ eran heterosexuales (1). Estos datos corroboran que la transmisión del virus de la inmunodeficiencia humana (VIH) ocurre principalmente por contacto sexual (2).

Aunque las mujeres representaron $110(28,2 \%)$ de los 390 casos de sida, cabe notar que la mayoría de los casos femeninos se notificaron en los últi- 
mos 2 años del período examinado (26 en 1991 y 53 en 1992). En general, la tasa anual de incidencia de sida por millón de habitantes guyaneses aumentó de 35,8 casos en 1988 a 83,2 en 1992, mientras que la razón de hombre a mujer se redujo de 3,5:1 a 1,6:1 en el mismo período. De los 265 pacientes masculinos y 102 femeninos cuya edad se registró en ese período, $80,8 \%$ de los primeros y $85,3 \%$ de los segundos pertenecían al grupo de 20 a 39 años de edad (1).

Para 1992, la Oficina de Estadística de Guyana había calculado una población nacional de 717458 habitantes (compuesta en 50,8\% de mujeres y en $49,2 \%$ de hombres), de los cuales $31,1 \%$ residían en zonas urbanas. La Región 4, una de las 10 regiones geopolíticas del país (donde se ubica Georgetown, la capital), tenía una población total de 297162 habitantes, 126004 de ellos $(42,4 \%)$ originarios de las Indias orientales y $142226(47,9 \%)$ de descendencia africana. De la población femenina de la Región 4, 31,9\% pertenecía al grupo de 15 a 29 años y $12,1 \%$ al de 30 a 39 (3).

Se ha demostrado una gran variabilidad mundial de la seroprevalencia del VIH en trabajadoras sexuales que ejercen la prostitución. Esa variabilidad ha guardado relación con factores tales como la edad de la trabajadora y su grado de escolaridad, estado civil, movilidad, número de clientes, tiempo de dedicación al oficio y precio, así como con sus patrones de uso de condones y antecedentes de enfermedades de transmisión sexual (ETS) y de abuso de drogas (4-14). Sin embargo, los resultados de las investigaciones efectuadas en trabajadoras sexuales han dado pie a la recomendación general de ofrecer a esas mujeres actividades educativas y otras actividades orientadas a prevenir la infección por el VIH $(15,16)$.

En algunos estudios sobre la seroprevalencia del VIH en trabajadoras sexuales se ha descubierto una sobreestimación del número de prostitutas de la calle y se ha postulado que diferencias en sus condiciones laborales y en la organización del trabajo podrían influir en la magnitud del riesgo al que se exponen esas mujeres. En términos más generales, varios problemas metodológicos y logísticos pueden frustrar la obtención de una muestra representativa de la población de trabajadoras sexuales, por lo que existe la posibilidad de un sesgo muestral en varios estudios realizados en trabajadoras sexuales atendidas en dispensarios médicos (14).

En Guyana se prohíbe la comercialización del sexo y de las drogas de uso recreativo y es natural, como se señalara anteriormente, que la conexión entre ambas actividades ejerza una importante influencia en la epidemiología y prevención de la infección por el VIH $(17,18)$.

En época reciente, las trabajadoras sexuales de Georgetown han sido perseguidas por la policía, que ha intentado expulsarlas de las calles de la ciudad. Quizá por tal motivo, las que ofrecen sus servicios públicamente en Georgetown tienden a acudir a lugares específicos: un grupo trabaja en ciertos hoteles, otro se reúne en determinadas calles, un tercer grupo trabaja en los bares y un cuarto grupo en Puerto Georgetown, principal puerto del país y sitio de acceso a naves visitantes.

Nuestro estudio reveló que las mujeres congregadas en ciertas calles solían cobrar las tarifas más bajas y tener la peor situación socioeconómica, siendo ello un posible reflejo de la situación socioeconómica de sus clientes regulares. Por consiguiente, al terminar la presente encuesta estas trabajadoras de la calle se clasificaron dentro de un estrato "inferior", y las que trabajaban en bares, hoteles y el puerto (que solían cobrar tarifas más altas) dentro de uno "superior".

El objetivo primordial de la presente investigación fue obtener información básica sobre la seroprevalencia del VIH en el grupo estudiado, con objeto de aplicarla en futuros estudios comparados y de obtener información de utilidad para actividades educativas sobre la salud. La falta de datos empíricos sobre el uso de drogas ilícitas en Guyana, sumada a la estrecha relación observada en otros estudios entre su uso y la seroprevalencia del VIH en trabajadoras sexuales, llevó a la solici- tud de información sobre el uso de drogas ilícitas (10).

La encuesta (realizada en abril de 1993) se hizo con el objetivo más concreto de reunir datos que permitieran estimar la prevalencia del VIH en las trabajadoras sexuales de Georgetown; que revelaran la posible influencia de su estado socioeconómico en la prevalencia del VIH, y que también proporcionaran información sobre sus conocimientos, actitudes, creencias y prácticas en relación con el VIH y el sida.

\section{MATERIALES Y MÉTODOS}

El estudio, realizado durante las semanas segunda y tercera de abril de 1993 (incluido un sábado por la noche) comenzó con visitas preliminares a los habituales sitios de reunión de las trabajadoras sexuales. Ahí se entró en contacto con las mujeres que estaban presentes, a quienes se explicó la finalidad de la encuesta y se pidió que instaran a sus colegas a participar. También se pidió la cooperación de los administradores y dueños de hoteles y bares, a quienes se aseguró que la encuesta no estaba vinculada en modo alguno con la policía. Se consiguió la colaboración de las autoridades de salud de Puerto Georgetown, quienes instaron a las trabajadoras sexuales del puerto a participar.

Se dio a las trabajadoras la fecha y la hora (después de las 19,00 h) de las visitas propuestas a determinados lugares, procurándose cumplir con el horario fijado. En dos localidades, las mujeres pidieron que se repitieran las visitas para que las colegas que estaban ausentes pudieran participar. Las visitas se repitieron tal como pidieron las mujeres.

Se explicó a las trabajadoras que la encuesta era anónima e independiente y que en ella se les harían varias preguntas y se les tomaría una muestra de sangre. A cambio de ello, cada una recibiría G\$ 500 (US\$ 4,00) y un estuche con artículos para el cuidado de la

\footnotetext{
5 G\$ 100 equivalían a cerca de US\$ 0,80 en el momento de la encuesta.
} 
salud en que había unos 10 condones y folletos sobre el VIH, el sida y otras ETS. El pago se hizo con la intención de indemnizar a las participantes por el tiempo laboral perdido y de reducir el número de rechazos. En lo sucesivo, ninguna de las trabajadoras, gerentes de hoteles o dueños de bares rehusó nuestra solicitud de cooperación en la encuesta, a excepción de una sola trabajadora que se negó a participar.

Se observó que las trabajadoras sexuales que abordaban a los clientes en la calle solían cobrar las tarifas más bajas por sus servicios (aproximadamente G\$500), y que las de los hoteles cobraban de G\$500 a G\$1 500 y las de los bares y el puerto, G\$ 1500 o más. Como se señalara anteriormente, siendo estas las tarifas y siendo las trabajadoras de la calle de más baja condición socioeconómica que las demás, se decidió clasificarlas en un estrato "inferior" y a las integrantes del otro grupo en uno "superior".

Las visitas preliminares a los sitios en que solían reunirse las trabajadoras sexuales revelaron que cerca de 500 trabajaban abiertamente en la ciudad. De ahí que se optara por continuar la encuesta hasta haber entrevistado a una muestra mínima de 100 . No se fijó un número límite de entrevistas por estrato y se dejó participar a toda mujer que lo deseara voluntariamente, procurándose no entrevistar a la misma persona en más de una ocasión. La posibilidad de doble participación se redujo mediante la realización de una sola visita a la mayor parte de las localidades, ya que casi todas las mujeres trabajaban únicamente en sitios específicos. En los lugares que se visitaron más de una vez (a solicitud de las propias trabajadoras), las mujeres mismas identificaron a las colegas que habían estado ausentes durante la visita anterior. Las mujeres que mostraban una natural tendencia a asumir un papel directivo ayudaron a evitar que hubiera entrevistas repetidas.

Para cubrir los hoteles y Puerto Georgetown, se asignaron ciertos lugares a tres entrevistadores previamente adiestrados (KC, BH y MJ) para que efectuaran las entrevistas. Para la cobertura de las calles y bares donde había trabajadoras, se empleó un autobús que también proporcionó un lugar apropiado para las entrevistas. Cada cuestionario aplicado en ellas se había codificado y adherido a un tubo de ensayo con el mismo código para la muestra de sangre de la participante. Los códigos del cuestionario y del tubo de ensayo correspondiente estaban cubiertos, de tal manera que no eran del conocimiento ni del entrevistador ni de la participante.

Después de completar el cuestionario aplicado para la entrevista, se envió a cada participante al técnico encargado de la encuesta (DN), que sacó a cada mujer una muestra de sangre de $10 \mathrm{~mL}$ y le entregó una remuneración de G\$500 más los condones o el estuche ya descrito para el cuidado de la salud. Los tubos de ensayo con las muestras de sangre se mantuvieron cerrados y en posición vertical en una rejilla a una temperatura promedio de $26^{\circ} \mathrm{C}$ al aire libre. Después de la toma de sangre, a cada participante se le dio información sobre la transmisión y prevención de la infección por el VIH, el sida y las ETS; se le explicó que los resultados de la prueba detectora del VIH no se entregarían individualmente, y se le aconsejó que acudiera a la Clínica de Medicina Genitourinaria del Hospital Público de Georgetown, donde podría obtener orientación, hacerse pruebas para la detección del VIH y otras ETS, recibir sus resultados, y conseguir condones gratuitamente.

Al final de cada actividad nocturna, las muestras de sangre recolectadas se llevaron al laboratorio. Ahí se sometieron a centrifugación y los sueros sobrantes se colocaron en almacenamiento. El tiempo máximo transcurrido entre la toma de la sangre de una participante y la colocación del suero en un refrigerador fue cercano a 6 horas. Posteriormente las muestras de suero se analizaron en el Laboratorio Nacional de Enfermedades Infecciosas mediante un inmunoensayo enzimático (ELISA) de marca Wellcozyme para la detección de VIH 1 y 2, más la prueba de inmunofluorescencia (IFA) para confirmar los diagnósticos positivos (19). Para el manejo de los datos se empleó el programa Epi-Info, que sir- vió para procesar y analizar la información recogida en el cuestionario y los resultados de la prueba detectora del VIH de cada participante.

\section{RESULTADOS}

Se identificó y reunió a un total de 109 trabajadoras sexuales en los diversos puntos donde se realizó la encuesta. Una trabajadora se negó a participar, por lo que el total se redujo a 108. La distribución de esas 108 participantes según su positividad al VIH, su estrato socioeconómico y 19 características investigadas se presenta en el cuadro 1.

En total, 27 de las 108 participantes (25\%; IC $95 \%=16,8$ a $33,2 \%)$ resultaron seropositivas al VIH. La seroprevalencia mucho más alta del VIH observada en el estrato inferior ( $42,0 \%$ en comparación con $10,3 \%$ en el superior) fue estadísticamente significativa (razón de posibilidades $(\mathrm{RP})=6,3$; IC $95 \%=$ 2,1 a 20,87 ).

Ninguna participante tenía menos de 15 años ni más de 39 y la mayoría pertenecían al grupo de 15 a 29; hubo mujeres positivas al VIH en ambos grupos de edad. Las diferencias de seropositividad al VIH no mostraron significación estadística cuando se analizaron por grupo de edad (15-29 frente a 30-39). Sin embargo, las 38 participantes del estrato inferior entre las edades de 15 y 29 años constituyeron dos terceras partes de las mujeres positivas al VIH. Como era de esperar, su tasa de seropositividad al VIH fue muy distinta de la de las participantes del estrato superior, bien fueran del grupo más joven $(\mathrm{RP}=6,3$; IC95\% $=$ $1,8$ a 24,5$)$ o del grupo de mayor edad $(\mathrm{RP}=15,3 ; \mathrm{IC} 95 \%=1,9$ a 674,2$)$.

Asimismo, los datos sobre estado civil no revelaron diferencias significativas en la seroprevalencia del VIH antes de ser desglosados por estratos. Sin embargo, las participantes de estrato bajo que declararon tener una relación estable (pasada o presente) mostraron una seroprevalencia del VIH mucho mayor que las mujeres del mismo estrato que declararon no tener ninguna relación estable $(\mathrm{RP}=7,7$; 
CUADRO 1. Resultados de la encuesta de trabajadoras sexuales realizada en Georgetown, Guyana, en abril de 1993, que muestra la seroprevalencia del VIH en participantes de diversas características, según su estrato. Las cifras de las tres columnas de la derecha representan el número de trabajadoras sexuales seropositivas al VIH en relación con el total de trabajadoras, el porcentaje de mujeres positivas y el intervalo de confianza de $95 \%$ (IC95\%) correspondiente a ese porcentaje

\begin{tabular}{|c|c|c|c|}
\hline Característica & Estrato inferior & Estrato superior & Total \\
\hline \multicolumn{4}{|l|}{ Grupo de edad (en años) } \\
\hline $30-39$ & $3 / 12(25,0 \% ; 0,5 \%-49,5 \%)$ & $1 / 18(5,6 \% ; 0,0 \%-16,2 \%)$ & $4 / 30(13,3 \% ; 1,2 \%-25,5 \%)$ \\
\hline \multicolumn{4}{|l|}{ Estado civil } \\
\hline Casada y en unión consensual & $14 / 27$ (51,9\%; 32,9\%-70,9\%) & $0 / 15(0,0 \%)$ & $14 / 42(33,3 \% ; 19,0 \%-47,6 \%)$ \\
\hline \multicolumn{4}{|l|}{ Grado de escolaridad de la trabajadora sexual } \\
\hline Ninguno/escuela primaria & $9 / 27(33,3 \% ; 15,6 \%-51,1 \%)$ & $3 / 35$ ( $8,6 \% ; 0,0 \%-17,9 \%)$ & $12 / 62(19,4 \% ; 9,5 \%-29,2 \%)$ \\
\hline Más de escuela primaria & $12 / 23(52,2 \% ; 31,8 \%-72,6 \%)$ & $3 / 23(13,0 \% ; 0,0 \%-26,8 \%)$ & $15 / 46(32,6 \% ; 19,1 \%-46,2 \%)$ \\
\hline \multicolumn{4}{|l|}{ No. de clientes a la semana } \\
\hline$\leq 5$ & $2 / 4(50,0 \% ; 1,0 \%-99,0 \%)$ & $3 / 37$ ( $8,1 \% ; 0,0 \%-16,9 \%)$ & $5 / 41(12,2 \% ; 2,2 \%-22,2 \%)$ \\
\hline$>5$ & $19 / 46(41,3 \% ; 27,1 \%-55,6 \%)$ & $3 / 21(14,3 \% ; 0,0 \%-29,3 \%)$ & $22 / 67(32,8 \% ; 21,6 \%-44,1 \%)$ \\
\hline \multicolumn{4}{|l|}{ Lugar donde se ofrece el servicio } \\
\hline Solo en Georgetown & $15 / 35$ (42,9\%; $26,5 \%-59,3 \%)$ & $6 / 34(17,6 \% ; 4,8 \%-30,4 \%)$ & $21 / 69(30,4 \% ; 19,6 \%-41,3 \%)$ \\
\hline En Georgetown y otras partes de Guyana & $3 / 5(60,0 \% ; 17,1 \%-99,9 \%)$ & $0 / 6(0,0 \%)$ & $3 / 11(27,3 \% ; 0,9 \%-53,6 \%)$ \\
\hline En Georgetown y en el exterior & $3 / 10(30,0 \% ; 1,6 \%-58,4 \%)$ & $0 / 18(0,0 \%)$ & $3 / 28(10,7 \% ; 0,0 \%-22,2 \%)$ \\
\hline \multicolumn{4}{|l|}{ Solicitud del uso de condones por los clientes } \\
\hline Nunca u ocasionalmente & $3 / 9$ (33,3\%; 2,5\%-64,1\%) & $1 / 7(14,3 \% ; 0,0 \%-40,2 \%)$ & $4 / 16(25,0 \% ; 14,2 \%-35,8 \%)$ \\
\hline Casi siempre o siempre & $18 / 41(43,9 \% ; 28,7 \%-59,1 \%)$ & $5 / 51(9,8 \% ; 1,6 \%-18,0 \%)$ & $23 / 92(25,0 \% ; 20,5 \%-29,5 \%)$ \\
\hline \multicolumn{4}{|l|}{ Posesión de condones por la trabajadora sexual } \\
\hline Nunca u ocasionalmente & $12 / 29(41,4 \% ; 23,5 \%-59,3 \%)$ & $4 / 22(18,2 \% ; 2,1 \%-34,3 \%)$ & $16 / 51(31,4 \% ; 24,9 \%-37,9 \%)$ \\
\hline Casi siempre o siempre & $9 / 21(42,9 \% ; 21,7 \%-64,0 \%)$ & $2 / 36(5,6 \% ; 0,0 \%-13,1 \%)$ & $11 / 57(19,3 \% ; 14,1 \%-24,5 \%)$ \\
\hline \multicolumn{4}{|l|}{ Uso de condones por los clientes guyaneses } \\
\hline Nunca u ocasionalmente & $5 / 16(31,3 \% ; 8,5 \%-54,0 \%)$ & $2 / 19(10,5 \% ; 0,0 \%-24,3 \%)$ & $7 / 35(20,0 \% ; 13,2 \%-26,8 \%)$ \\
\hline \multicolumn{4}{|l|}{$\begin{array}{l}\text { Relaciones sexuales sin condón a pedido } \\
\text { del cliente, si la trabajadora está necesitada }\end{array}$} \\
\hline Sí & $7 / 18(38,9 \% ; 16,4 \%-61,4 \%)$ & $2 / 28(7,1 \% ; 0,0 \%-16,6 \%)$ & $9 / 46(19,6 \% ; 13,7 \%-25,5 \%)$ \\
\hline No & $14 / 32(43,8 \% ; 26,6 \%-60,9 \%)$ & $4 / 30(13,3 \% ; 1,1 \%-25,5 \%)$ & $18 / 62(29,0 \% ; 23,2 \%-34,8 \%)$ \\
\hline \multicolumn{4}{|l|}{$\begin{array}{l}\text { Preferiría perder a los clientes que se nieguen } \\
\text { a usar condón }\end{array}$} \\
\hline Sí & $20 / 43(46,5 \% ; 31,6 \%-61,4 \%)$ & $4 / 34(11,8 \% ; 1,0 \%-22,6 \%)$ & $24 / 77(31,2 \% ; 25,9 \%-36,5 \%)$ \\
\hline No & $1 / 7(14,3 \% ; 0,0 \%-40,2 \%)$ & $2 / 24(8,3 \% ; 0,0 \%-19,3 \%)$ & $3 / 31(9,7 \% ; 4,4 \%-15,0 \%)$ \\
\hline \multicolumn{4}{|l|}{ Conocimiento de la causa/transmisión del sida } \\
\hline Respuesta apropiada & $3 / 4(75,0 \% ; 32,6 \%-99,9 \%)$ & $0 / 11(0,0 \%)$ & $3 / 15(20,0 \% ; 9,7 \%-30,3 \%)$ \\
\hline Respuesta inapropiada & $18 / 46(39,1 \% ; 25,0 \%-53,2 \%)$ & $6 / 47(12,8 \% ; 3,3 \%-22,3 \%)$ & $24 / 93(25,8 \% ; 21,3 \%-30,3 \%)$ \\
\hline \multicolumn{4}{|l|}{ Antecedentes de ETS en los 2 años anteriores } \\
\hline Sí & $7 / 16(43,8 \% ; 19,4 \%-68,1 \%)$ & $1 / 11(9,1 \% ; 0,0 \%-26,1 \%)$ & $8 / 27(29,6 \% ; 20,8 \%-38,4 \%)$ \\
\hline No & $14 / 34(41,2 \% ; 24,6 \%-57,8 \%)$ & $5 / 47(10,6 \% ; 1,8 \%-19,4 \%)$ & $19 / 81(23,5 \% ; 18,8 \%-28,2 \%)$ \\
\hline \multicolumn{4}{|l|}{ Se considera expuesta al riesgo de contraer VIH } \\
\hline Sí & $10 / 25(40,0 \% ; 20,8 \%-59,2 \%)$ & $4 / 40(10,0 \% ; 4,5 \%-15,5 \%)$ & $14 / 65(21,5 \% ; 16,4 \%-26,6 \%)$ \\
\hline No & $11 / 25(44,0 \% ; 24,5 \%-63,5 \%)$ & $2 / 18(11,1 \% ; 0,0 \%-25,6 \%)$ & $13 / 43(30,2 \% ; 23,2 \%-37,2 \%)$ \\
\hline
\end{tabular}


CUADRO 1. (Continuación)

\begin{tabular}{lccc}
\hline \multicolumn{1}{c}{ Característica } & Estrato inferior & Estrato superior & Total \\
\hline $\begin{array}{l}\text { Razones de la falta de temor en torno al riesgo } \\
\text { de infección por el VIH }\end{array}$ & & & \\
Religiosas & $0 / 2(0,0 \%)$ & $0 / 0(0,0 \%)$ & $0 / 2(0,0 \%)$ \\
Uso de condones & $7 / 16(43,8 \% ; 19,4 \%-68,1 \%)$ & $0 / 7(0,0 \%)$ & $7 / 23(30,4 \% ; 20,8 \%-40,0 \%)$ \\
Suerte & $3 / 4(75,0 \% ; 32,6 \%-99,9 \%)$ & $1 / 5(20,0 \% ; 0,0 \%-55,1 \%)$ & $4 / 9(44,4 \% ; 27,8 \%-61,0 \%)$ \\
No se dio ninguna razón & $1 / 3(33,3 \% ; 0,0 \%-86,7 \%)$ & $1 / 6(16,7 \% ; 0,0 \%-46,5 \%)$ & $2 / 9(22,2 \% ; 8,3 \%-36,1 \%)$ \\
Uso de cocaína & & & \\
Sí & $6 / 8(75,0 \% ; 45,0 \%-99,9 \%)$ & $0 / 4(0,0 \%)$ & $6 / 12(50,0 \% ; 35,6 \%-64,4 \%)$ \\
No & $15 / 42(35,7 \% ; 21,2 \%-50,2 \%)$ & $6 / 54(11,1 \% ; 2,7 \%-19,5 \%)$ & $21 / 96(21,9 \% ; 16,5 \%-27,3 \%)$ \\
Sabe de la disponibilidad gratuita de condones & & \\
Sí & $17 / 37(45,9 \% ; 29,9 \%-62,0 \%)$ & $0 / 30(0,0 \%)$ & $17 / 67(25,4 \% ; 20,1 \%-30,7 \%)$ \\
No & $4 / 13(30,8 \% ; 5,7 \%-55,9 \%)$ & $6 / 28(21,4 \% ; 6,2 \%-36,6 \%)$ & $10 / 41(24,4 \% ; 17,7 \%-31,1 \%)$ \\
\hline
\end{tabular}

IC $95 \%=1,4$ a 77,5), o que las mujeres de estrato superior que declararon haber tenido antes o tener en ese momento una relación estable (RP: 10,7; IC95\% = 2,8-48,5) o no tener ninguna (RP = 9,5; IC95\% = 1,7 a 93,7).

Tampoco se observó una relación general entre la seropositividad al VIH y el grado de escolaridad (solo una participante del estrato más alto dijo que no tenía instrucción formal), pero se determinó una seroprevalencia mucho mayor del VIH en mujeres de estrato más bajo, independientemente de la escolaridad. Los datos concretos fueron los siguientes: mujeres con instrucción primaria solamente, estrato inferior frente a estrato superior: $\mathrm{RP}=$ 5,3, IC95\% = 1,11 a 33,6; mujeres con estudios posprimarios, estrato inferior frente a estrato superior: $\mathrm{RP}=7,3$, IC95\% = 1,5 a 46,6; y mujeres de estrato inferior con estudios posprimarios frente a mujeres de estrato superior con instrucción primaria solamente: $\mathrm{RP}=11,6, \mathrm{IC} 95 \%=2,4$ a 72,5 .

Casi todas las participantes del estrato inferior pero relativamente pocas del estrato superior declararon que recibían a más de cinco clientes semanales. En general, la tasa de prevalencia del VIH de las mujeres que declararon más de cinco clientes a la semana fue mucho mayor que la de las mujeres que declararon menos clientes $(\mathrm{RP}=3,5 ; \mathrm{IC} 95 \%=1,1$ a 12,9$)$.

No se observó ninguna diferencia estadística significativa en la seroprevalencia del VIH de mujeres que habían ejercido la prostitución por más de 12 meses o por menos. No obstante, casi todas las participantes de estrato social bajo (seis de siete) que declararon haber ejercido la prostitución por menos de 12 meses salieron positivas al VIH. En este grupo la seroprevalencia del VIH fue mucho mayor que entre las 43 participantes de estrato bajo con más experiencia ( $\mathrm{RP}=$ 11,$2 ;$ IC95\% = 1,1 a 533,0), que entre las 20 de estrato alto con menos de 12 meses de experiencia $(\mathrm{RP}=34,0$; IC $95 \%=2,3$ a 1650,7$)$, y que entre las 38 de estrato alto con más experiencia.

Tampoco se observó ninguna diferencia estadística significativa entre las mujeres que trabajaban solo en Georgetown y las que trabajaban en otros lugares. (Suriname, país vecino, y las islas del Caribe de habla francesa, holandesa e inglesa fueron algunos de los lugares extranjeros notificados.) No obstante, todas las mujeres seropositivas del estrato superior declararon haber trabajado en Georgetown exclusivamente. Además, la ya citada diferencia estadísticamente significativa de seroprevalencia observada entre las mujeres de estrato inferior y superior se tradujo en una diferencia estadísticamente significativa en la seroprevalencia del VIH entre las mujeres de estrato inferior y superior que declararon haber trabajado en Georgetown solamente $(\mathrm{RP}=3,5 ; \mathrm{IC} 95 \%=1,0$ a 12,8).

En lo que respecta al uso de condones, $92(85,2 \%)$ de las participantes declararon que lo exigían a sus clientes muy a menudo o invariablemente. Sin embargo, no se observó ninguna diferencia estadísticamente significativa entre la prevalencia del VIH en ese grupo de mujeres y en el de las mujeres que dijeron que nunca exigían el uso de condones o que solo lo exigían en ocasiones. Cincuenta y siete (53\%) de las participantes dijeron que disponían de condones frecuentemente o siempre; 54 de 89 (61\%) indicaron su uso frecuente o invariable entre sus clientes guyaneses y 61 de 66 (92\%) entre sus clientes extranjeros. Como posible resultado del tamaño de la muestra, no se encontraron diferencias estadísticamente significativas en la prevalencia del VIH entre mujeres de similar estrato que diferían en sus patrones de uso de condones.

Al compararse la seroprevalencia del VIH según la nacionalidad declarada de los clientes, se encontró que la seropositividad al VIH era mucho mayor entre mujeres que atendían solo a clientes guyaneses que entre las que tenían clientes extranjeros exclusivamente $(\mathrm{RP}=9,0$; IC95\% = 1,1 a 401,3). Sin embargo, la seropositividad general al VIH entre las mujeres cuyos clientes eran guyaneses y extranjeros no fue muy diferente de la hallada entre mujeres que tenían solamente clientes guyaneses.

En general, 46 (42\%) de las participantes del estudio (36\% de las de estrato bajo y $48 \%$ de las de estrato alto) admitieron que estaban dispues- 
tas a tener relaciones sexuales sin condón a solicitud del cliente y que necesitaban dinero con urgencia. Sin embargo, 77 (71\%) mujeres, 86\% de ellas pertenecientes al estrato más bajo y $59 \%$ al estrato más alto, dijeron que preferían perder a un cliente que se negaba categóricamente a usar condón. Cabe señalar que la seropositividad al VIH fue mucho mayor entre esas 77 mujeres que entre las 31 tendientes a quedarse con el cliente $(\mathrm{RP}=$ 4,$2 ;$ IC $95 \%=1,1$ a 23,6 ).

Solo 15 participantes $(13,9 \%)$ sabían que el sida era consecuencia de la infección por el VIH y conocían por lo menos dos de las vías de transmisión del virus. Tres de las cuatro participantes de estrato inferior que poseían estos conocimientos salieron positivas al VIH, a diferencia de ninguna de las 11 mujeres pertenecientes al estrato más alto que también los poseían $(\mathrm{RP}=4,4 ; \mathrm{IC} 95 \%=1,4$ a 15,0$)$.

En lo que respecta a otras ETS, no hubo diferencia estadísticamente significativa en la seroprevalencia del VIH entre mujeres con y sin antecedentes declarados de ETS en los 2 años anteriores.

Sesenta y cinco $(60 \%)$ de las participantes dijeron sentirse expuestas al riesgo de contraer la infección por el VIH y $43(40 \%)$ dijeron no sentirse expuestas a ese riesgo. De estas últimas, 34 dieron una variedad de explicaciones, algunas alusivas al uso de condones y otras al azar o a la religión (cuadro 1).

Doce de las trabajadoras sexuales $(11,1 \%)$ notificaron el uso de cocaína. Aunque la seropositividad al VIH fue mayor entre ellas, la diferencia global no fue estadísticamente significativa $(P$ exacta de Fisher $=0,0694 ;$ IC95\% $=$ 0,85 a 14,72). Dos terceras partes de las mujeres que notificaron usar cocaína pertenecían al estrato inferior.

En general, 67 (62\%) de las participantes dijeron estar enteradas de la disponibilidad de condones gratuitos en la Clínica de Medicina Genitourinaria y en la Asociación de Guyana para la Paternidad Reponsable. Las diferencias de seroprevalencia entre mujeres con y sin este conocimiento no fue estadísticamente significativa. Casi tres cuartas partes $(74 \%)$ de las mujeres pertenecientes al estrato inferior dijeron poseer esta información, en comparación con solo $52 \%$ de las que pertenecían al estrato superior.

En el cuadro 2 se proporcionan datos sobre la prevalencia del VIH entre las 28 participantes que dijeron usar marihuana o cocaína. En los dos grupos de edad estudiados (15-29 y 30-39 años), una buena fracción del más joven ( 24 mujeres de un total de 78 , frente a 4 de un total de 30 ) admitieron el uso de marihuana o cocaína.
No obstante, las consumidoras de marihuana o cocaína pertenecientes al grupo de mayor edad tuvieron una tasa más alta de seropositividad que las que no usaban estas drogas, y la diferencia fue estadísticamente significativa $(\mathrm{RP}=75,0 ; \mathrm{IC} 95 \%=2,2 \mathrm{a}$ $4074,4)$.

Como puede apreciarse en los cuadros 3 y 4, un mayor porcentaje de las mujeres pertenecientes al estrato superior dijeron estar dispuestas a tener relaciones sexuales sin el uso de un condón, en comparación con las que

CUADRO 2. Resultados de la encuesta que muestran la seroprevalencia del VIH en las participantes que declararon que usaban cocaína o marihuana o que no habían usado ninguna de las dos, por grupo de edad. Las cifras de las dos columnas de la derecha representan el número de trabajadoras sexuales seropositivas al VIH en relación con el total de trabajadoras, y el porcentaje de seropositivas

\begin{tabular}{ccc}
\hline $\begin{array}{c}\text { Grupo de edad } \\
\text { (en años) }\end{array}$ & $\begin{array}{c}\text { Mujeres que declararon } \\
\text { el uso de marihuana, } \\
\text { cocaína, o ambas }\end{array}$ & $\begin{array}{c}\text { Mujeres que declararon } \\
\text { no usar ni marihuana } \\
\text { ni cocaína }\end{array}$ \\
\hline $15-29$ & $6 / 24(25,0 \%)$ & $17 / 54(31,5 \%)$ \\
$30-39$ & $3 / 4(75,0 \%)$ & $1 / 26(3,8 \%)$ \\
Total & $9 / 28(32,1 \%)$ & $18 / 80(22,5 \%)$ \\
\hline
\end{tabular}

CUADRO 3. Resultados de la encuesta, con el número de participantes que manifestaron estar dispuestas a tener relaciones sexuales sin condón, según tiempo de servicio y estrato. Las cifras de las tres columnas de la derecha representan el número de trabajadoras sexuales que declararon estar dispuestas a tener relaciones sexuales sin condón en relación con el total de trabajadoras, y el porcentaje de mujeres dispuestas a tenerlas

\begin{tabular}{crcr}
\hline Tiempo de servicio & Estrato inferior & Estrato superior & \multicolumn{1}{c}{ Total } \\
\hline$\leq 12$ meses & $1 / 7(14,3 \%)$ & $9 / 20(45 \%)$ & $10 / 27(37,0 \%)$ \\
$>12$ meses & $17 / 43(39,5 \%)$ & $19 / 38(50 \%)$ & $36 / 81(44,4 \%)$ \\
Total & $18 / 50(36,0 \%)$ & $28 / 58(48,3 \%)$ & $46 / 108(42,6 \%)$ \\
\hline
\end{tabular}

CUADRO 4. Resultados de la encuesta, con el número de participantes que declararon estar dispuestas a tener relaciones sexuales sin condón, según estrato y antecedentes de enfermedades de transmisión sexual (ETS) en los 2 años anteriores a la encuesta. Las cifras en las tres columnas de la derecha representan el número de trabajadoras sexuales que declararon estar dispuestas a tener relaciones sexuales sin condón en comparación con el total de trabajadoras, y el porcentaje de mujeres dispuestas a tenerlas

\begin{tabular}{lrrr}
\hline $\begin{array}{l}\text { Antecedentes } \\
\text { de ETS }\end{array}$ & Estrato inferior & Estrato superior & \multicolumn{1}{c}{ Total } \\
\hline Sí & $9 / 16(56,3 \%)$ & $7 / 11(63,6 \%)$ & $16 / 27(59,3 \%)$ \\
No & $9 / 34(26,5 \%)$ & $21 / 47(44,7 \%)$ & $30 / 81(37,0 \%)$ \\
Total & $18 / 50(36,0 \%)$ & $28 / 58(48,3 \%)$ & $46 / 108(42,6 \%)$ \\
\hline
\end{tabular}


pertenecían al estrato inferior $(48 \%$ y $36 \%$, respectivamente). Asimismo, un mayor porcentaje de las que dijeron haber tenido ETS en los 2 años anteriores (comparadas con las que no notificaron ETS en ese período) dijeron que estarían dispuestas a tener relaciones sexuales sin condón.

\section{DISCUSIÓN Y CONCLUSIONES}

La seroprevalencia del VIH detectada en las mujeres que participaron en el estudio fue de $25 \%$, con un IC $95 \%$ de 16,8 a 33,2\%. Ello indica que, si estas mujeres fueron representativas de todas las trabajadoras sexuales de Georgetown, de una sexta a una tercera parte de estas últimas estarían infectadas por el VIH.

Huelga señalar que la disposición a participar en el estudio podría haber sido resultado de la insistencia de los gerentes de hoteles y dueños de bares, de la buena relación con las autoridades de sanidad portuaria y de la presión ejercida por las compañeras de trabajo. Asimismo, el pago a las trabajadoras (remuneración por el tiempo gastado) también podría haber producido cierto sesgo de selección, ocasionado especialmente por la solicitud de algunas participantes del estrato más bajo de que se repitiera la visita del entrevistador para asegurar la participación de sus colegas. Cabe señalar también el pequeño número de mujeres que integraron la muestra en algunas categorías como resultado de la estratificación. En general no hay motivos para suponer que los resultados sean aplicables a todas las trabajadoras sexuales de Guyana; pero es razonable concluir que proporcionan información útil sobre la percepción del riesgo, las conductas riesgosas y la seroprevalencia del VIH entre las mujeres que trabajan en Georgetown.

Con una sola excepción, todas las participantes, que tenían más de 15 años, habían cursado estudios de primaria. Este resultado coincide con las estadísticas nacionales, según las cuales el grado de alfabetismo en Guyana se aproxima a $90 \%$. En lo que respecta al origen étnico, 37\% de las participan- tes eran de origen africano, $37 \%$ de origen indio oriental, $10,2 \%$ de origen amerindio y $15,8 \%$ de raza mixta. Como se estima que la población amerindia representa un porcentaje mínimo de la población de Guyana, el hecho de que más de $10 \%$ de las prostitutas estudiadas fueran amerindias apunta a las dificultades particulares que enfrenta este grupo a la hora de prepararse para conseguir y desempeñar empleos remunerados cuando llegan a la ciudad de zonas del interior, donde no son iguales las oportunidades educativas ni de otra índole.

Se detectaron infecciones por el VIH en mujeres pertenecientes a todos los grupos étnicos estudiados. Más de $60 \%$ de las participantes habían nacido en la Región de Demerara-Mahaica, donde se sitúa la ciudad de Georgetown; pero la población estudiada también se compuso de mujeres nacidas en todas las 10 regiones de Guyana. Se determinó que las mujeres positivas al VIH procedían de la zona de Demerara-Mahaica y de otras de cuatro zonas.

En general, las participantes que cobraban tarifas más bajas tenían un mayor número de clientes semanales y la tasa de positividad al VIH observada mostró una tendencia a aumentar con el número de clientes. Aunque el uso de condones se considera importante, poco más de la mitad de las participantes tenían condones para sus clientes. Al mismo tiempo, los clientes guyaneses suelen usar condones con menos frecuencia que los extranjeros y tienen una mayor tendencia a transmitir el virus o a infectarse. Por ende, en Guyana se necesita una iniciativa educativa mucho más intensa para erradicar el mito de que el virus es propagado principalmente por extranjeros. Después de años de discusión, se espera que la población entienda que el uso de condones reduce el riesgo de transmisión del VIH. Sin embargo, también se palpa la gran necesidad de intensificar la promoción y comercialización de condones en Guyana.

La seroprevalencia del VIH fue menor en trabajadoras sexuales con más tiempo de servicio, situación contraria en cierta medida a la que se encontró en otro estudio en Nairobi, Kenya, entre prostitutas de estratos socioeconómicos más bajos, en que se observó mayor seropositividad en mujeres que habían sido prostitutas por más tiempo (4). En Georgetown, esto podría reflejar una relación directa entre más años de experiencia en la práctica de la prostitución y una mayor autoestima o conocimiento profesional, como sugiere el hecho de que las mujeres que habían ejercido la prostitución por menos tiempo estaban más dispuestas a prestar servicios aunque el cliente no quisiera usar condón.

Casi todas las mujeres que participaron en el estudio temían perder a los clientes si les exigían el uso de condones; sin embargo, las mujeres con antecedentes de ETS fueron las más propensas a aceptar tener relaciones sexuales sin condón.

La seroprevalencia del VIH fue un poco menor ( 22 frente a $30 \%$ ) entre las trabajadoras sexuales que se sentían expuestas al riesgo de infección por el VIH que entre las que no tenían ese temor. Aunque la diferencia no fue estadísticamente significativa, la situación sugiere que las personas que se sienten preocupadas pueden ser más proclives a tomar precauciones que las que no se preocupan. Asmismo, cabe tener presente la posibilidad de que las participantes hayan dado contestaciones tendientes a complacer a los entrevistadores.

En general, las participantes tenían conocimientos poco satisfactorios sobre el sida y la transmisión del VIH. Solamente $22(20,4 \%)$ sabían que acualmente no hay ninguna cura conocida para personas con sida.

Aunque casi todas las participantes declararon haberse convertido en prostitutas por motivos económicos, muchas admitieron a los entrevistadores que usaban drogas ilícitas. Por lo tanto, podría haber una relación entre la práctica de la prostitución y la satisfacción de la necesidad de consumir drogas.

En general, menos mujeres del estrato superior sabían de la distribución gratuita de condones en la Clínica de Medicina Genitourinaria y en la Asociación de Guyana para la Paterni- 
dad Responsable, actividad ignorada por todas las mujeres seropositivas del estrato más alto. Es posible que la falta de conocimiento de las mujeres del estrato superior acerca de la disponibilidad de condones se debiera al uso exclusivo del servicio de salud pública gratuito por mujeres del estrato más bajo.

También cabe señalar que algunas de las mujeres de estrato más alto que habían viajado al exterior se habían visto obligadas a presentarse al sistema de salud pública (de algunos territorios extranjeros donde habían trabajado) para sacar un permiso médico. La experiencia podría relacionarse en cierta medida con la obtención de información sobre la distribución gratuita de condones en Guyana. En términos más generales, varias participantes opinaron que debían estar matriculadas, ser sometidas a exámenes médicos ocasionales y recibir un carné de sanidad que les permitiera trabajar abiertamente, como se acostumbra en algunos países vecinos.

Podría ser necesario hacer enmiendas a la ley de comercialización del sexo. En el entretiempo, sin embargo, la transmisión activa del VIH y la subsiguiente aparición de casos de sida en Guyana han creado una urgente necesidad de acción por parte de las trabajadoras sexuales y sus clientes. En otras palabras, es preciso hacer un esfuerzo concertado por promover la modificación del comportamiento, mejorar la disponibilidad y accesibilidad de condones, atender a los casos de ETS, explicar claramente las medidas para prevenir la infección por el VIH y las ETS, y hacer que el público cobre mayor conciencia de la magnitud del problema constituido por la infección por el VIH, el sida y las ETS en el país mediante la provisión de información apropiada y oportuna.
Agradecimiento. Los autores agradecen a Lloyd Godette, a Carol Trotman y al personal del Laboratorio Nacional de Enfermedades Infecciosas de Georgetown el análisis de las muestras en el laboratorio; a Peter Carr (Representante), Fred Nunes y el fallecido Eliécer Peña, funcionarios de la OPS en Georgetown, la revisión del documento y su apoyo durante el estudio; a Paloma Cuchí de la OPS en Washington, su generosa colaboración; a Indra Beharry-Lall su ayuda como mecanógrafa y a Wilton Bailey su contribución como conductor del autobús en Georgetown. Agradecemos en especial a los funcionarios del Ministerio de Salud de Guyana y a las trabajadoras sexuales que participaron en el estudio, sin cuya buena voluntad y colaboración no habría sido posible realizarlo. El costo del estudio fue sufragado por el Programa Nacional del Sida de Guyana.

\section{REFERENCIAS}

1. Pan American Health Organization/World Health Organization. AIDS surveillance in the Americas. Washington, DC: PAHO/WHO; diciembre de 1992. (Informe cuatrimestral).

2. Mann J. Global AIDS: epidemiology, impact, projections, global strategy. En: World Health Organization. AIDS prevention and control. Geneva: WHO; 1988:3-14.

3. Guyana, Bureau of Statistics. Guyana Stat Bull 1992:5.

4. Simonsen JN, Plummer FA, Ngugi EN, et al. HIV infection among lower socioeconomic strata prostitutes in Nairobi. AIDS 1990;4: 139-144.

5. McKeganey N, Barnard M. Selling sex: female street prostitution and HIV risk behaviour in Glasgow. AIDS-Care 1992;4(4):395-407.

6. Hernández-Aguado I, Fernández-García E, García de la Hera M, Alvarez-Dardet C. Infección por el virus de la inmunodeficiencia humana tipo 1 en prostitutas y factores de riesgo. Med Clin Barc 1992;99(11):406-409.

7. Rehle T, Brinkmann UK, Siraprapsiri T, Coplan P, Aiemsukawat C, Ungchusak K. Risk factors of HIV 1 infection among female prostitutes in Khon Kaen, Northeast Thailand. Infection 1992;20(6):328-331.
8. Gwede C, McDermott RJ. AIDS in sub-Saharan Africa: implications for health education. AIDS Educ Prev 1992;4(4):350-361.

9. Wong ML, Tan TC, Ho ML, Lim JY, Waan S, Chan R. Factors associated with sexually transmitted diseases among prostitutes in Singapore. Infect J STD-AIDS 1992;3(5): 332-337.

10. Modan B, Goldschmidt R, Rubinstein E, Vonsover A, et al. Prevalence of HIV antibodies in transsexual and female prostitutes. Am J Public Health 1992;82(4):590-592.

11. Pickering H, Todd J, Dunn D, et al. Prostitutes and their clients: a Gambian survey. Soc Sci Med 1992;34(1):75-88.

12. Wynall FS, Hyams KC, Phillips IA, et al. Sexual transmission of human T-lymphotrophic virus type 1 in Peruvian prostitutes. $J$ Med Virol 1992;38(1):44-48.

13. Plourde PJ, Plummer FA, Pepin J, et al. Human Immunodeficiency virus type 1 infection in women attending a sexually transmitted disease clinic in Kenya. I Infect Dis 1992;166(1):86-92.

14. Jackson L, Highcrest A, Coates RA. Varied potential risks of HIV infection among prostitutes. Soc Sci Med 1992;35(3):281-286.
15. Anónimo. Street outreach for STD/HIV prevention, Colorado Springs, Colorado, 19871991. MMWR 1992;41(6):94-101.

16. Dorfman LE, Derish PA, Cohen JB. Hey girlfriend: an evaluation of AIDS prevention among women in the sex industry. Health Educ Q 1992;19(1):25-40.

17. Day S. Editorial review: prostitute women and AIDS (anthropology). AIDS 1988;2: 421-428.

18. Padian N. Editorial review: prostitute women and AIDS (epidemiology). AIDS 1988;2: 413-419.

19. George JR, Schochetman G. Detection of HIV infection using serologic techniques. En: Schochetman G, George JR, eds. AIDS testing: a comprehensive guide to technical, medical, social, legal, and management issues. New York: Springer Verlag; 1994:62-102.

Manuscrito recibido el 9 de febrero de 1995 y aceptado para publicación en versión revisada el 14 de noviembre de 1996 
ABSTRACT A study of 108 female sex workers engaged in prostitution in Georgetown, Guyana, was made in April 1993. Based on interviews and procurement of blood samples, the study investigated relationships between HIV seroprevalences and AIDS knowledge, risk behaviors, client characteristics, and condom use. Street-walkers-as distinct from sex workers in bars, hotels, and Port Georgetown-tended to charge less, be worse off socioeconomically, and have clients who were similarly disadvantaged; they were therefore classified as belonging to a "lower" socioeconomic stratum, while the other workers were classified as belonging to a "higher" stratum. The overall HIV seroprevalence found among the sex workers was 25\% (95\%CI: 17\%-33\%). But the 50 subjects in the lower stratum had a relatively high seroprevalence $(42 \%$, as compared to $10 \%$ among those in the higher stratum), accounting for 21 of the 27 HIV-seropositive subjects. Reported patterns of client origins (Guyanese or foreign), worker willingness to have sex without a condom, and condom use by clients differed by stratum. Participants in the higher stratum were more disposed to having sex without a condom. The workers' knowledge of what causes AIDS and how HIV is transmitted was low in both strata; substantial numbers of workers said they had contracted a sexually transmitted disease within the past two years or were users of illicit drugs. Condom use is reportedly less common among Guyanese than foreign clients, suggesting a greater risk of contracting HIV from Guyanese clients or infecting Guyanese clients with it. The HIV seroprevalence among workers who said they had only Guyanese clients was statistically greater than the rate among those who said they had only foreign clients. The HIV seroprevalence among those reporting more than five clients per week was statistically greater than among those reporting fewer. HIV seropositivity was relatively high among the 12 workers who said they used cocaine. Overall, the findings supported the view that interventions targeted at female sex workers and their clients should be strengthened-more specifically, that concerted efforts should be made to intensify condom promotion, distribution, and social marketing; to improve STD services that provide treatment and counseling for female sex workers; and to increase educational activities among the workers' Guyanese clients.

\title{
20. ${ }^{\circ}$ seminario anual "Adelantos en traumatología"
}

Fechas: 12 a 13 de diciembre de 1997

Lugar: Hotel Ritz-Carlton, Kansas City, Misuri, Estados Unidos de América

Tema: Técnicas actualizadas en traumatología

Este seminario, patrocinado por el Comité de Traumatología del Colegio Estadounidense de Cirujanos para la Región VII (Iowa, Kansas, Misuri y Nebraska) como parte de un proyecto de educación médica continuada, contará con la participación de eminentes expertos en el campo de la traumatología. En él se presentan los últimos adelantos en el manejo de casos complejos, la movilización del paciente gravemente herido y el uso de tecnologías modernas para la evaluación del paciente de trauma.

Aunque los participantes pueden inscribirse en el propio seminario, es aconsejable que lo hagan por anticipado. El costo es de US\$ 350 para médicos, US\$ 200 para personal de enfermería de atención crítica, y US\$150 para médicos residentes. Si se desea asistir al seminario un solo día, el costo se reduce en 60\%. Toda inscripción posterior al 10 de noviembre de 1997 tendrá un recargo de US\$ 25.

\author{
Información: \\ Ms. Carol Williams \\ American College of Surgeons \\ Trauma Department \\ 55 East Erie Street \\ Chicago, Illinois 60611, USA \\ Teléfono: (312) 665 4050, ext. 342 \\ Correo electrónico: cwilliams@facs.org
}

